\title{
In situ Transmission Electron Microscopy of Copper Electrodeposition
}

\author{
F. M. Ross ${ }^{+}$, M. J. Williamson*, R. M. Tromp ${ }^{+}$, R. Hull ${ }^{*}$ and P. M. Vereecken ${ }^{+}$
}

${ }^{+}$IBM T. J. Watson Research Center, Yorktown Heights, NY 10598

"School of Engineering and Applied Science, University of Virginia, Charlottesville, VA 22903

Real time, in situ microscopy of surface and interface processes at low pressure has been very successful: it has allowed us to determine reaction mechanisms, observe transient structures and measure the parameters which control growth. Processes which take place in liquid environments are equally interesting scientifically and technologically but have not been as straightforward to observe in situ [1]. One important liquid process is the electrochemical deposition of copper, used in fabricating interconnects in advanced integrated circuits. To optimize the final structure it is useful to observe the nucleation of $\mathrm{Cu}$ clusters and to follow the growth of clusters to coalescence. In this paper we describe a simple cell in which we can control and observe this process in the TEM.

The cell is shown in figure 1. We etch two SiN-covered $\mathrm{Si}$ wafers to form windows and then glue them together with the windows aligned. An oxide layer maintains a separation of $0.5-1 \mu \mathrm{m}$ between the wafers. On one wafer we deposit an electron transparent Au working electrode partly over the viewing window and connect it electrically through the wafer to an external contact. The counter electrode is a Au wire extending into one of the reservoirs. The reservoirs, capped with glass spacers and sealed with sapphire lids [2], are designed to make it easier to introduce the electrolyte with a syringe. $\mathrm{A} \mathrm{CuSO} / 4 / \mathrm{H}_{2} \mathrm{SO}_{4}$ electrolyte is used. To improve the image quality, images are energy filtered and recorded digitally at 12 frames per second [3].

Figure 2 shows stills from a video recorded during $\mathrm{Cu}$ deposition and figure 3 illustrates the kinetics of cluster growth. Nucleation is clearly progressive rather than instantaneous, but the process is rapid with all clusters nucleating within 3 seconds. Progressive nucleation followed by diffusionlimited growth has been inferred indirectly from current transient measurements $[4,5]$. Our real time data allows us to measure the initial nucleation rate $\left(2.5 \times 10^{8} \mathrm{~cm}^{-2} \mathrm{sec}^{-1}\right.$, within the range cited in literature [5]), and a linearly increasing nucleus density as expected theoretically [5]. After nucleation, we find that the growth of individual clusters can be understood with a simple electrochemical model [6].

It is important to consider the limitations arising from the finite volume of electrolyte available above the electrode. Voltammograms recorded in the cell are similar to those made in a standard three-electrode electrochemical cell, suggesting that the same process is taking place. However, we find that at higher current densities deposition becomes limited by diffusion of $\mathrm{Cu}$ ions from distant regions of the electrolyte. Calculations suggest that we can reproduce the bulk process if we choose appropriate length and time scales, and that the early stages of nucleation and growth are not too sensitive to the limited volume provided a low growth rate is used [6]. We can even extend the range of the experiments by applying the voltage in pulses. The liquid cell we have described may also be adapted to study phenomena such as corrosion, liquid crystal switching and biological systems, where the ability to analyze the solid/liquid interface in real-time and with reasonable resolution will help us gain a deeper understanding of the important processes involved [7]. 
[1] P. L. Gai, Microsc. Microanal. 8 (2002) 21.

[2] We used a heat curing epoxy (Measurements Group Inc., Raleigh, NC) to glue the wafers together and a UV-cured epoxy (Summers Optical, Fort Washington, PA) to glue the sapphire lids after introducing the electrolyte.

[3] Imaging energy filter and video rate CCD camera supplied by Gatan, Inc., Pleasanton, CA [4] M. H. Hölzle et al., Electrochim. Acta 40 (1995), 1237.

[5] G. Gunawardena, G. Hills, I. Montenegro and B. Scharifker, J. Electroanal. Chem. 138 (1982) 225; P. M. Vereecken, K. Strubbe and W. P. Gomes, J. Electroanal. Chem. 433 (1997) 19; A. Radisic et al., J. Electrochem. Soc. 148 (2001) C41.

[6] M. J. Williamson, R. M. Tromp, R. Hull, P. M. Vereecken and F. M. Ross, in preparation.

[7] We acknowledge S. J. Chey, M. C. Reuter, A. Ellis P. C. Searson, J. Horkens, R. G. Kelly and J. M. Harper for their contributions.

FIG 1: Components of the electrochemical cell. The viewing window is enlarged for clarity.

FIG 2: Images extracted from a video recorded during deposition of copper at a constant current density of $5 \mathrm{mAcm}^{-2}$. The scale bar is 1 micron. Images are shown after $0,0.7,1.3,2.7$ and 4.7 seconds.
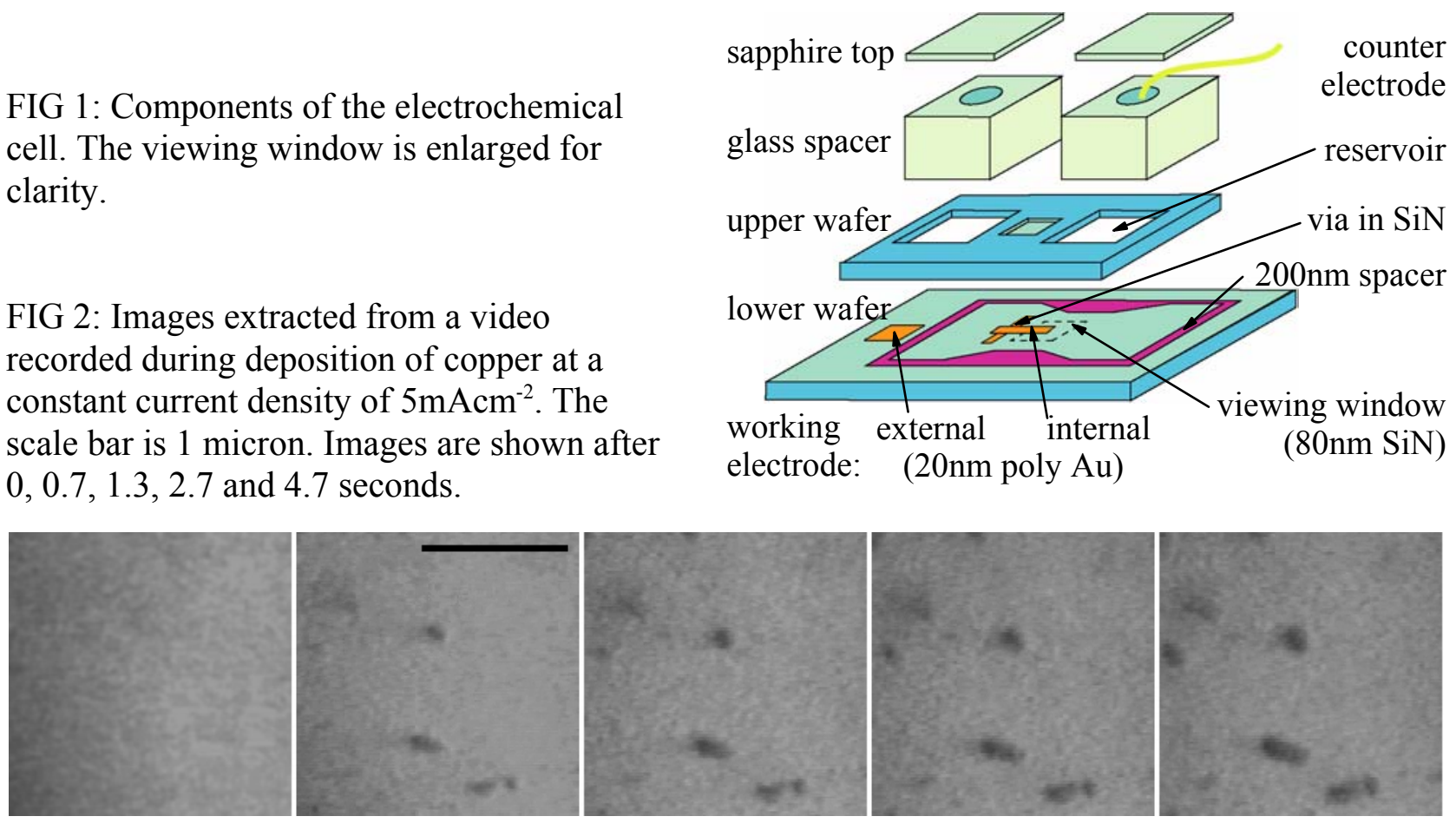

FIG 3: (a) Number density of nuclei obtained from Fig. 2. The density initially increases linearly as expected. (b) Average $\mathrm{Cu}$ thickness, calculated assuming nuclei are hemispherical (as seen in ex situ SEM). The straight line shows the expected deposition rate. The volume flattens out as ions are depleted from the liquid above the electrode. (c) Growth of individual clusters during

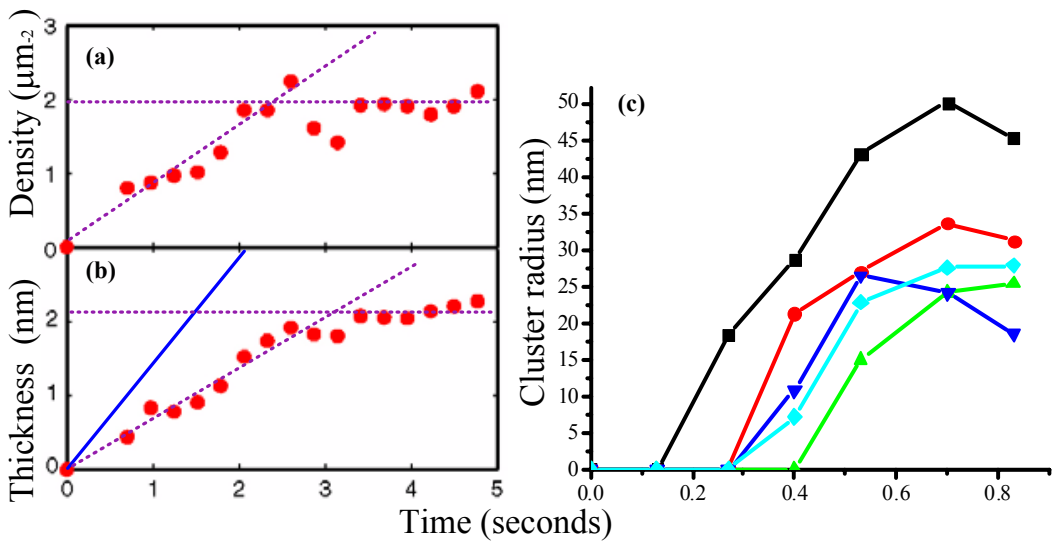
deposition at $50 \mathrm{mAcm}^{-2}$. 